\title{
Immersion and Invariance-Based Coordinated Generator Excitation and SVC Control for Power Systems
}

\author{
Adirak Kanchanaharuthai \\ Department of Electrical Engineering, Rangsit University, Pathumthani 12000, Thailand \\ Correspondence should be addressed to Adirak Kanchanaharuthai; adirak@rsu.ac.th
}

Received 24 July 2013; Revised 16 January 2014; Accepted 18 January 2014; Published 5 March 2014

Academic Editor: Yuri Vladimirovich Mikhlin

Copyright (c) 2014 Adirak Kanchanaharuthai. This is an open access article distributed under the Creative Commons Attribution License, which permits unrestricted use, distribution, and reproduction in any medium, provided the original work is properly cited.

\begin{abstract}
A nonlinear coordinated control of excitation and SVC of an electrical power system is proposed for transient stability, and voltage regulation enhancement after the occurrence of a large disturbance and a small perturbation. Using the concept of Immersion and Invariance (I\&I) design methodology, the proposed nonlinear controller is used to not only achieve power angle stability, frequency and voltage regulation but also ensure that the closed-loop system is transiently and asymptotically stable. In order to show the effectiveness of the proposed controller design, the simulation results illustrate that, in spite of the case where a large perturbation occurs on the transmission line or there is a small perturbation to mechanical power inputs, the proposed controller can not only keep the system transiently stable but also simultaneously accomplish better dynamic properties of the system as compared to operation with the existing controllers designed through a coordinated passivation technique controller and a feedback linearization scheme, respectively.
\end{abstract}

\section{Introduction}

As a result of power systems with the rapid increase of the size and complexity, power system stability, including power angle stability as well as frequency and voltage regulation, is of great importance. In general, the stability margins of the power system decrease as the electrical power transmission levels increase. It is well known that recently the power system operation is faced with the difficult task of maintaining stability when small or large disturbances occur in the power system. Therefore, more effective and efficient control methodologies for improvement of power system stability are desired. In particular, great attention has been paid to the design of an advanced nonlinear controller design to not only improve power system stability margins but also enhance controllability and increase power transfer capability in the literature for years.

Although there have been numerous studies for improving power system stability, recently an effective approach to improving the stability of power systems uses generator excitation control in combination with Flexible AC Transmission Systems (FACTS) devices. FACTS devices
$[1,2]$ are becoming increasingly important for improving the controllability of power flows and voltages as well as the stability of the power systems. FACTS devices include SVC, STATCOM, TCSC, SSSC, TCPAR, and UPFC, and these devices are often employed in interconnected and long-distance transmission systems to improve power flow, voltage control, interarea and system oscillations, reactive power control, and steady-state and dynamic stability. In this paper, among a family of these FACTS devices, Static Var Compensator (SVC) is of interest since it is used in power systems to regulate the system voltage and improve power system stability: in particular it is capable of rapidly injecting and absorbing active and reactive power in order to increase grid transfer capability through enhanced dynamic voltage stability, to provide smooth and rapid reactive power compensation for voltage support, and to improve both damping oscillations and transient stability [3-7].

To the best of our knowledge, although considerable research has addressed the application of SVC, less attention has been devoted to the coordination of generator excitation and SVC controller for power systems through the nonlinear control theory. In [8], the SVC controller was proposed using 
the exact linearization method. In [9], using the feedback linearization method and control of differential and algebraic systems, a coordinated generator excitation and SVC controller in power systems with nonlinear loads was proposed and it can improve the power angle stability of generators and the voltage behavior. In [10], a nonlinear controller design for SVC to improve power system voltage stability using direct feedback linearization technique was proposed. In [11], based on adaptive and robust control technique, the SVC controller was proposed to enhance power system voltage stability. More recently, in [12], based on modifying adaptive backstepping sliding model control methodology an adaptive backstepping sliding mode $\mathscr{H}_{\infty}$ controller was proposed for static var compensator alone. The controller not only attenuates the influences of external disturbances on the system output but also has strong robustness for system parameter variations.

This paper continues this line of investigation and improves further transient response performance by using a different technique based on the concept of Immersion and Invariance (I\&I) for the design of a nonlinear control law for transient stability and voltage regulation enhancement of power systems with SVC. Recently, relatively little prior work using I\&I technique has been devoted to the combination of generator excitation and other FACTS such as STATCOM [13] and CSC [14]. Nevertheless, note that the resulting controllers are under the assumption of full state feedback. This to not leads only an important drawback but also further increases a difficulty in control design procedure due to practically immeasurable states and particularly generator internal transient voltage sources.

In this work, the assumption can be directly relaxed by using all measurable states as state variables instead of unmeasurable ones in the whole dynamic models. Therefore, the proposed controller not only achieves power angle stability along with frequency and voltage regulation but also keeps the system transiently stable. Simulation results are provided for a single machine infinite bus (SMIB) power system with excitation control of a synchronous generator and SVC control.

The rest of this paper is organized as follows. In Section 2, the problem formulation is provided. In Section 3, power system models used are briefly given. The I\&I design method used to construct a nonlinear control law is stated in Section 4. In Section 5, simulation results are given. Finally, we conclude in Section 6.

\section{Problem Formulation}

In this paper, we are interested in studying the system stability enhancement of a nonlinear power system including generator excitation controller and SVC. The nonlinear system considered can be written in the general form as follows:

$$
\dot{x}(t)=f(x)+g(x) u(x),
$$

where $x \in \mathbb{R}^{n}$ is the state vector, $u \in \mathbb{R}^{m}$ is the control action, and $f(x)$ and $g(x)$ are assumed to be smooth functions.

The problem of interest in this paper is the following: given a stable equilibrium point $x_{e}$, find a stabilizing feedback

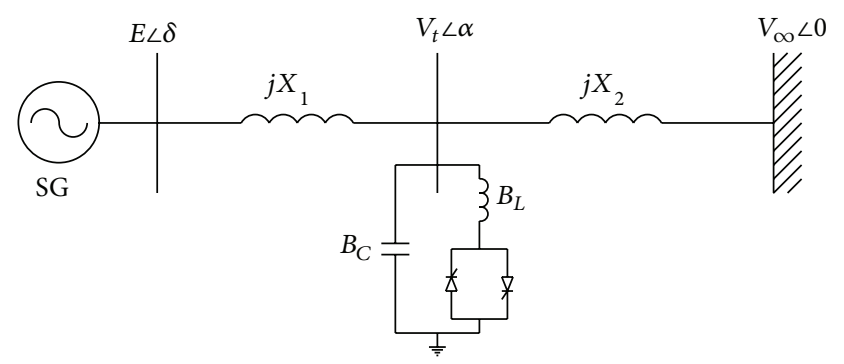

FIGURE 1: Network with TCR-FC SVC.

controller $u(x)$ so that the closed-loop system satisfies the following.

(1) The desired equilibrium point $x_{e}$ is asymptotically and transiently stable.

(2) Power angle stability, voltage, and frequency regulation are simultaneously achieved.

In the next section, we mention simplified nonlinear models of power systems and use these to design the stabilizing feedback control law that meets the requirements (1)-(2) given above.

\section{Power System Models}

The power system models considered here consist of the dynamics of a synchronous generator and SVC. A dynamic model of the synchronous generator (SG) can be obtained by representing the SG by a transient voltage source, $E$, behind a transient reactance, $X_{d}^{\prime}$. In this paper, as depicted in Figure 1, a thyristor-controlled-reactor (TCR) fixed-capacitor type of SVC is employed and the SVC can serve as a variable susceptance connected in shunt (parallel) with the power systems [12]. For simplicity, the dynamic behavior of the SVC is often modeled as a first-order differential equation. As a result, the dynamics of synchronous generator with excitation control and the SVC regulator in SMIB power systems can be modeled as follows [10]:

$$
\begin{gathered}
\dot{\delta}=\omega-\omega_{s}, \\
\dot{\omega}=\frac{1}{M}\left(P_{m}-P_{E}-D\left(\omega-\omega_{s}\right)\right), \\
\dot{E}=-\frac{X_{d \Sigma}}{X_{d \Sigma}^{\prime} T_{0}^{\prime}} E+\frac{X_{d}-X_{d}^{\prime}}{X_{d \Sigma}^{\prime} T_{0}^{\prime}} V_{\infty} \cos \delta+\frac{u_{f}}{T_{0}^{\prime}}, \\
\dot{B}_{L}=\frac{1}{T_{r}}\left(B_{L}-B_{L 0}+u_{r}\right)
\end{gathered}
$$

with

$$
P_{E}=\frac{E V_{\infty} \sin \delta}{X_{d \Sigma}^{\prime}-\left(X_{d}^{\prime}+X_{T}\right) X_{L}\left(B_{L}-B_{C}\right)},
$$

where $\delta$ is the power angle of the generator, $\omega$ denotes the relative speed of the generator, $D \geq 0$ is a damping constant, $P_{E}$ is the electrical power delivered by the generator to the 
voltage at the infinite bus $V_{\infty}, \omega_{s}$ is the synchronous machine speed, $\omega_{s}=2 \pi f, H$ represents the per unit inertial constant, $f$ is the system frequency, and $M=2 H / \omega_{s} . X_{d \Sigma}^{\prime}=X_{d}^{\prime}+$ $X_{T}+X_{L}$ is the reactance consisting of the direct axis transient reactance of $S G$, the reactance of the transformer, and the reactance of the transmission line $X_{L}$. Similarly, $X_{d \Sigma}=X_{d}+$ $X_{T}+X_{L}$ is identical to $X_{d \Sigma}^{\prime}$ except that $X_{d}$ denotes the direct axis reactance of SG. $T_{0}^{\prime}$ is the $d$-axis transient short-circuit time constant. $X_{1}=X_{d}^{\prime}+X_{T}, X_{2}=X_{L}, u_{f}$ is the field voltage control input. $P_{m}$ is the mechanical input power to be assumed constant throughout this paper. $B_{L}$ and $B_{C}$ are the susceptance of the inductor in SVC (pu) and the equivalent capacitor (pu.), $B_{L 0}$ is the initial value of the inductor in SVC (pu.), $u_{r}$ is the SVC control input to be designed, and $T_{r}$ is a SVC time constant.

In practice, the generator transient voltage $(E)$ is often physically not measurable and $B_{L}-B_{C}$ may not be convenient to monitor as active electrical power; thus, the active power $P_{E}$ can be divided into two new variables, namely, an active electrical power of generation excitation alone $P_{e}$ and a real electrical power of the SVC device $P_{\mathrm{svc}}$, as follows:

$$
\begin{gathered}
P_{E}=P_{e}+P_{\mathrm{svc}}, \\
P_{e}=\frac{E V_{\infty} \sin \delta}{X_{d \Sigma}^{\prime}} \\
P_{\mathrm{svc}}=\frac{\left(X_{d}^{\prime}+X_{T}\right) X_{L}\left(B_{L}-B_{C}\right)}{X_{d \Sigma}^{\prime}-\left(X_{d}^{\prime}+X_{T}\right) X_{L}\left(B_{L}-B_{C}\right)} \frac{E V_{\infty} \sin \delta}{X_{d \Sigma}^{\prime}} \\
=\frac{\left(X_{d}^{\prime}+X_{T}\right) X_{L}\left(B_{L}-B_{C}\right)}{X_{d \Sigma}^{\prime}-\left(X_{d}^{\prime}+X_{T}\right) X_{L}\left(B_{L}-B_{C}\right)} P_{e} .
\end{gathered}
$$

Differentiating both state variables and defining the state variables $x_{1}=\delta, x_{2}=\omega-\omega_{s}, x_{3}=P_{e}$, and $x_{4}=P_{\mathrm{svc}}$, the dynamic model of power systems including SVC can be expressed as the general form (1) as follows:

$$
\begin{aligned}
& f(x)=\left[\begin{array}{l}
f_{1}(x) \\
f_{2}(x) \\
f_{3}(x) \\
f_{4}(x)
\end{array}\right] \\
&= {\left[\begin{array}{c}
x_{2} \\
\frac{1}{M}\left(P_{m}-D x_{2}-x_{3}-x_{4}\right) \\
\left(-a+x_{2} \cot x_{1}\right) x_{3}+\frac{b V_{\infty} \sin 2 x_{1}}{X_{d \Sigma}^{\prime}} \\
\widetilde{M}\left(x_{1}, x_{3}, x_{4}\right) f_{3}(x)+\widetilde{N}\left(x_{1}, x_{3}, x_{4}\right)\left(B_{L}-B_{L 0}\right)
\end{array}\right], } \\
& x=\left[\begin{array}{c}
x_{1} \\
x_{2} \\
x_{3} \\
x_{4}
\end{array}\right],
\end{aligned}
$$

$$
\begin{aligned}
& g(x)=\left[\begin{array}{cc}
0 & 0 \\
0 & 0 \\
g_{31}(x) & 0 \\
g_{41}(x) & g_{42}(x)
\end{array}\right] \\
&= {\left[\begin{array}{cc}
0 & 0 \\
0 & 0 \\
\frac{V_{\infty} \sin x_{1}}{X_{d \Sigma}^{\prime}} & 0 \\
\widetilde{M}\left(x_{1}, x_{3}, x_{4}\right) g_{31}(x) & -\widetilde{N}\left(x_{1}, x_{3}, x_{4}\right)
\end{array}\right], } \\
& u(x)=\left[\begin{array}{l}
u_{f} \\
T_{0}^{\prime} \\
\frac{u_{r}}{T_{r}}
\end{array}\right],
\end{aligned}
$$

where

$$
\begin{gathered}
\widetilde{M}\left(x_{1}, x_{3}, x_{4}\right)=\left(\frac{X_{d \Sigma}^{\prime}}{X_{d \Sigma}^{\prime}-\left(X_{d}^{\prime}+X_{T}\right) X_{L}\left(B_{L}-B_{C}\right)}-1\right), \\
\widetilde{N}\left(x_{1}, x_{3}, x_{4}\right)=-\frac{x_{3}\left(X_{d}^{\prime}+X_{T}\right) X_{L} X_{d \Sigma}^{\prime}}{\left(X_{d \Sigma}^{\prime}-\left(X_{d}^{\prime}+X_{T}\right) X_{L}\left(B_{L}-B_{C}\right)\right)^{2}} \\
B_{L}=\frac{1}{\left(X_{d}^{\prime}+X_{T}\right) X_{L}}\left(X_{d \Sigma}^{\prime}-\frac{x_{3} X_{d \Sigma}^{\prime}}{x_{3}+x_{4}}\right)+B_{C} \\
a=\frac{X_{d \Sigma}}{X_{d \Sigma}^{\prime} T_{0}^{\prime}}, \quad b=\frac{X_{d \Sigma}-X_{d \Sigma}^{\prime}}{X_{d \Sigma}^{\prime} T_{0}^{\prime}} V_{\infty} .
\end{gathered}
$$

Further, the bus (terminal) voltage $V_{t}$ as shown in Figure 1 can be found from the following expression:

$$
\begin{gathered}
V_{t}=\left(1+\frac{x_{4}}{x_{3}}\right) \cdot \frac{\Delta\left(x_{1}, x_{3}\right)}{X_{d \Sigma}^{\prime}}, \\
\Delta\left(x_{1}, x_{3}\right) \\
=\sqrt{\left(\frac{x_{3} X_{d \Sigma}^{\prime} X_{2}}{V_{\infty} \sin x_{1}}\right)^{2}+\left(V_{\infty} X_{1}\right)+2 X_{1} X_{2} X_{d \Sigma}^{\prime} x_{3} \cot x_{1} .}
\end{gathered}
$$

Additionally, the region of operation is defined in the set $\mathscr{D}=\left\{x \in \mathcal{S} \times \mathbb{R} \times \mathbb{R} \times \mathbb{R} \mid 0<x_{1}<\pi / 2\right\}$. The open loop operating equilibrium is denoted by $x_{e}=$ $\left[x_{1 e}, x_{2 e}, x_{3 e}, x_{4 e}\right]^{T}=\left[\delta_{e}, 0, P_{m}, 0\right]^{T}$.

From the dynamic equations in (5), it is easy to see that all state variables $\left(x_{1}, x_{2}, x_{3}, x_{4}\right)$ are measurable and such state variables can be used to find the bus voltage $V_{t}$. Therefore, the objective of this paper is to design a state feedback control law that meets the expected performance requirements (1)(2) given above.

Remark 1. Recently, there are numerous nonlinear control design techniques, such as feedback linearization scheme 
and control Lyapunov function. One of the most effective nonlinear control design techniques methods is a backstepping design [15] which is a constructive design method for nonlinear systems in a strict-feedback form. In (5), it is obvious that the dynamics of the state variable $\omega$ rely on not only the state variable $P_{e}$ but also the state variable $P_{\mathrm{svc}}$. Therefore, the dynamic equations in (5) are not the strictfeedback form. In contrast to the I\&I controller presented in this paper, backstepping design needs to be extended for the design of nonlinear controllers in the non-strict-feedback form; see [16] for further details.

Remark 2. It is well known that a large interconnected system can be usually represented by an infinite bus once its voltage and frequency remain constant under all conditions. In this work, based on the proposed controller, how power system stability of the coordination of a generator excitation and SVC can be enhanced, when connected to a large interconnected system or an infinite bus, is investigated. Even though in a large-scale power system there are several generators, it is often possible to reduce the system to a set of equivalent (one or two) machines that are of interest, connected to an equivalent network (Thevenin equivalent circuit) as shown in Figure 1. Roughly speaking, the SMIB power systems considered in this paper can be regarded as a subsystem of a multimachine power system. On the other hand, if the reduced order power system is not an adequate representation of the system for transient stability studies, then we can extend the control method proposed in this work to multimachine systems with SVC which will be reported in the future.

\section{Immersion and Invariance}

The I\&I method for stabilizing nonlinear systems was proposed in [17] and further developed as summarized in [18]. The method is based on the notion of invariant manifolds and system immersion. This methodology carries out from transforming the original state of the system $x(t)$ into two new states, namely, $\xi(t)$ and $z(t)$. The dimension of state $\xi(t)$ becomes strictly less than the dimension of state $x(t)$. The new reduced state $\xi(t)$ is called the target dynamics and the transformation employed to get these states defines the invariant manifold. The state $z(t)$ is called the off-themanifold state and complements the dimension of $\xi(t)$. The resulting control law is designed to ensure that the original state $x(t)$ is bounded, that the manifold is rendered invariant, and that the off-line-manifold state $z(t)$ converges asymptotically to the origin. Besides, the original state $x(t)$ will converge to a desired equilibrium point with a dynamic behavior converging to that of the target dynamical system. Roughly speaking, the I\&I concept relies upon selecting a target dynamical system that is capable of capturing the desired behavior of the closed-loop system to be controlled. The control objective of this method is to find a stabilizing control law that ensures that the closed-loop system behaves asymptotically the same as the prespecified target system, that is, achieves asymptotic model matching.
This method is applicable to practical control design problems for many types of systems; refer to [18] for further details. For transient stability and voltage regulation enhancement of power systems with excitation control, see $[19,20]$, with a controllable series capacitor (CSC), see [14], and with a static var compensator (STATCOM), see [13].

Based on the principle of Immersion and Invariance mentioned above, the following result [17] will be used for our nonlinear controller design.

Theorem 3 (see $[17,18]$ ). Consider the following nonlinear system; it is assumed that all functions and mapping are smooth, that is, $\mathbb{C}^{\infty}$, throughout this paper:

$$
\dot{x}(t)=f(x)+g(x) u(x),
$$

with state $x \in \mathbb{R}^{n}$ and control input $u \in \mathbb{R}^{m}$ and the vector fields $f: \mathbb{R}^{n} \rightarrow \mathbb{R}^{n}$ and $g: \mathbb{R}^{n} \rightarrow \mathbb{R}^{n \times m}$ being Lipschitz along with an assignable equilibrium point $x_{e} \in \mathbb{R}^{n}$ to be stabilized. Let $s<n$, and assume that there exist smooth mappings $\alpha$ : $\mathbb{R}^{s} \rightarrow \mathbb{R}^{s}, \pi: \mathbb{R}^{s} \rightarrow \mathbb{R}^{n}, c: \mathbb{R}^{n} \rightarrow \mathbb{R}^{m}, \phi: \mathbb{R}^{n} \rightarrow \mathbb{R}^{n-s}$, and $\varphi: \mathbb{R}^{n \times(n-s)} \rightarrow \mathbb{R}^{m}$, such that the following hold.

(H1) Target system. The system

$$
\dot{\xi}=\alpha(\xi)
$$

with state $\xi \in \mathbb{R}^{s}$ has an asymptotically stable equilibrium at $\xi_{e} \in \mathbb{R}^{s}$ and $x_{e}=\pi\left(\xi_{e}\right)$.

(H2) Immersion condition. For all $\xi \in \mathbb{R}^{s}$,

$$
f(\pi(\xi))+g(\pi(\xi)) c(\pi(\xi))=\frac{\partial \pi(\xi)}{\partial \xi} \alpha(\xi) .
$$

(H3) Implicit manifold. The set identity

$$
\begin{aligned}
\mathscr{M} & :=\left\{x \in \mathbb{R}^{n} \mid x=\pi(\xi) \text { for some } \xi \in \mathbb{R}^{s}\right\} \\
& =\left\{x \in \mathbb{R}^{n} \mid \phi(x)=0\right\}
\end{aligned}
$$

holds.

(H4) Manifold attractivity and trajectory boundedness. All trajectories of the system

$$
\begin{gathered}
\dot{z}=\frac{\partial \phi(x)}{\partial x}[f(x)+g(x) \varphi(x, z)], \\
\dot{x}=f(x)+g(x) \varphi(x, z)
\end{gathered}
$$

are bounded and satisfy

$$
\lim _{t \rightarrow \infty} z(t)=0 \text {. }
$$

Then, $x_{e}$ is a globally asymptotically stable equilibrium of the closed loop system

$$
\dot{x}=f(x)+g(x) \varphi(x, \phi(x)) .
$$

Taken from [18], Theorem 3 can be interpreted as follows. Given the nonlinear system (8) and the selected target 
dynamical system (9), find, if possible, a manifold $\mathscr{M}=\{x \in$ $\left.\mathbb{R}^{n} \mid x=\pi(\xi), \xi \in \mathbb{R}^{s}\right\}$ that can be rendered invariant and asymptotically stable and for which the restriction of the closed-loop system to $\mathscr{M}$ is described by the target system (H1). Note that the control input $u$ that makes the manifold invariant is not unique; it is only uniquely defined on $\mathscr{M}$; that is, $\varphi(\pi(\xi), 0)=c(\pi(\xi))$. According to (H4), in order to drive the off-the-manifold coordinate $z$ to zero and keep the system trajectories bounded, one of all possible controls is selected.

\subsection{I\&I Controller Design}

4.1.1. Target System. In order to design a stabilizing controller and verify the condition according to Theorem 3 , we start with selecting the target dynamics $(\dot{\xi}=\alpha(\xi))$ as the mechanical subsystems (e.g., a simple damped pendulum system):

$$
\begin{gathered}
\dot{\xi}_{1}=\xi_{2}, \\
\dot{\xi}_{2}=-\frac{\partial V\left(\xi_{1}\right)}{\partial \xi_{1}}-R(\xi) \xi_{2}
\end{gathered}
$$

where $V\left(\xi_{1}\right)$ and $R(\xi)$ represent the potential energy and a damping function of the pendulum systems, respectively, which both are to be selected. The pendulum system considered with a stable equilibrium point $\xi_{e}=\left(\xi_{1 e}, 0\right)^{T}$ has the potential energy $V\left(\xi_{i}\right)$ satisfying the following: (i) $\left(\partial V\left(\xi_{1 e}\right)\right) / \partial \xi=0$ and (ii) $\left(\partial^{2} V\left(\xi_{1 e}\right)\right) / \partial^{2} \xi>0$, the damping function verifying $R\left(\xi_{e}\right) \geq 0$, and the energy function $H(\xi)=$ $(1 / 2) \xi_{2}^{2}+V\left(\xi_{1}\right)$

4.1.2. Immersion Condition. As the desired target systems are selected, a mapping $\pi: \mathcal{S} \times \mathbb{R} \rightarrow \mathcal{S} \times \mathbb{R} \times \mathbb{R} \times \mathbb{R}$ is the following:

$$
\pi\left(\xi_{1}, \xi_{2}\right):=\left[\xi_{1}, \xi_{2}, \pi_{3}(\xi), \pi_{4}(\xi)\right]^{T}
$$

where $\pi_{3}(\xi)$ and $\pi_{4}(\xi)$ are to be selected. Besides, the condition of Theorem 3 gives the constraints, namely, $\xi_{1 e}=$ $x_{1 e}, \xi_{2 e}=x_{2 e}=0, \pi_{3}\left(\xi_{e}\right)=x_{3 e}=P_{m}$, and $\pi_{4}\left(\xi_{e}\right)=x_{4 e}=0$. We can choose $\pi_{3}(\xi)$ and $\pi_{4}(\xi)$ to satisfy the condition (10) as shown in

$$
\begin{aligned}
& {\left[\begin{array}{c}
\xi_{2} \\
\frac{1}{M}\left(P_{m}-\pi_{3}(\xi)-\pi_{4}(\xi)\right)-\frac{D}{M} \xi_{2} \\
f_{3}(\xi) \\
f_{4}(\xi)
\end{array}\right]} \\
& +\left[\begin{array}{cc}
0 & 0 \\
0 & 0 \\
g_{31}(\xi) & 0 \\
g_{41}(\xi) & g_{42}(\xi)
\end{array}\right]\left[\begin{array}{l}
c_{1}(\pi(\xi)) \\
c_{2}(\pi(\xi))
\end{array}\right]
\end{aligned}
$$

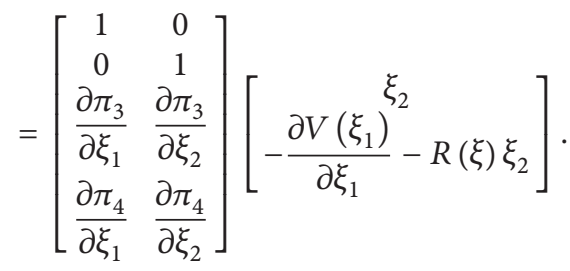

We choose the potential energy $V\left(\xi_{1}\right)$ satisfying two conditions (i)-(ii) mentioned previously as $V\left(\xi_{1}\right)=-\beta \cos \widetilde{\xi}_{1}$, $\tilde{\xi}_{1}=\xi_{1}-\xi_{1 e}$, for some $\beta>0$, and $R(\xi)=\left(D+\gamma_{d}\right) / M$.

Consider the second row; therefore, we have

$$
\begin{aligned}
\frac{1}{M}\left(P_{m}-\pi_{3}(\xi)-\pi_{4}(\xi)\right)-\frac{D}{M} \xi_{2} \\
=-\frac{\partial V\left(\xi_{1}\right)}{\partial \xi_{1}}-R(\xi) \xi_{2} \\
=-\beta \sin \left(\widetilde{\xi}_{1}\right)-\frac{D+\gamma_{d}}{M} \xi_{2}, \quad \gamma_{d} \geq 0 .
\end{aligned}
$$

From the expression in (17), in order to simplify our derivations, we choose $\pi_{4}\left(\xi_{2}\right)=x_{4 e}+\gamma_{d} \xi_{2}$. Consequently, we can compute $\pi_{3}(\xi)$ as follows:

$$
\pi_{3}(\xi)=P_{m}+\beta M \sin \widetilde{\xi}_{1}+\gamma_{d} \xi_{2}-\pi_{4}(\xi)
$$

It is obvious that $\pi_{3}$ is a function of both $\xi_{1}$ and $\xi_{2}$. As the mapping $\pi(\xi)$ has been chosen, by using some lengthy, but straightforward, calculations from the third and fourth rows, respectively, we have the control input $\left(u_{f} / T_{0}^{\prime}, u_{r} / T_{r}\right)^{T}=$ $\left(c_{1}(\pi(\xi)), c_{2}(\pi(\xi))\right)^{T}$ in (17) that renders the manifold $\mathscr{M}$ invariant.

4.1.3. Implicit Manifold. From the result above, the mapping $\pi(\xi)$ has been defined and the condition in (11) is verified. Therefore, the manifold $\mathscr{M}$ can be implicitly described by $\mathscr{M}=\{x \in \mathcal{S} \times \mathbb{R} \times \mathbb{R} \times \mathbb{R} \mid \phi(x)=0\}$ in which the mapping $\phi(x)$ can be defined as follows:

$$
\phi(x)=\left[\begin{array}{c}
x_{3}-\pi_{3}\left(x_{1}, x_{2}\right) \\
x_{4}-\pi_{4}\left(x_{2}\right)
\end{array}\right] .
$$

4.1.4. Manifold Attractivity and Trajectory Boundedness. In this subsection, a control law $u=\varphi(x, z)$ is designed to ensure that all trajectories of the closed-loop system are bounded and converge to the manifold $\mathscr{M}$. Let $z:=\phi(x)$ be the off-the-line 
manifold coordinate; substituting $\dot{x}_{3}, \dot{x}_{4}$ into the expressions below, then we have

$$
\begin{aligned}
\dot{z}_{1}= & \dot{x}_{3}-\dot{\pi}_{3}\left(x_{1}, x_{2}\right) \\
= & \left(-a+x_{2} \cot x_{1}\right) x_{3}+\frac{b V_{\infty} \sin 2 x_{1}}{2 X_{d \Sigma}^{\prime}} \\
& +\frac{V_{\infty} \sin x_{1}}{X_{d \Sigma}^{\prime}} \cdot \frac{\varphi_{1}(x, z)}{T_{f}}-\frac{\partial \pi_{3}}{\partial x_{1}} \dot{x}_{1}-\frac{\partial \pi_{3}}{\partial x_{2}} \dot{x}_{2}, \\
\dot{z}_{2}= & \dot{x}_{4}-\dot{\pi}_{4}\left(x_{2}\right) \\
= & f_{4}(x)+g_{31}(x) \cdot \frac{\varphi_{1}(x, z)}{T_{f}} \\
& +g_{42}(x) \cdot \frac{\varphi_{2}(x, z)}{T_{r}}-\frac{\partial \pi_{4}}{\partial x_{2}} \dot{x}_{2} .
\end{aligned}
$$

In order to ensure that the trajectories of the off-the-manifold coordinate $z$ are bounded and $\lim _{t \rightarrow+\infty} z(t)=0$ according to condition (13), we take $\dot{z}_{i}=-\gamma_{i} z_{i}, \gamma_{i}>0, i=1,2$ and then we can write

$$
\begin{gathered}
\frac{V_{\infty} \sin x_{1}}{X_{d \Sigma}^{\prime}} \cdot \frac{1}{T_{f}} \varphi_{1}(x, z) \\
=-\left(-a+x_{2} \cot x_{1}\right) x_{3}-\frac{b V_{\infty} \sin 2 x_{1}}{2 X_{d \Sigma}^{\prime}} \\
+\frac{\partial \pi_{3}}{\partial x_{1}} \dot{x}_{1}+\frac{\partial \pi_{3}}{\partial x_{2}} \dot{x}_{2}-\gamma_{1} z_{1}, \\
g_{42}(x) \cdot \frac{1}{T_{r}} \varphi_{2}(x, z)=-f_{4}(x)-g_{41}(x) \cdot \frac{\varphi_{1}(x, z)}{T_{f}} \\
+\frac{\partial \pi_{4}}{\partial x_{2}} \dot{x}_{2}-\gamma_{2} z_{2} .
\end{gathered}
$$

4.1.5. The Control Law. From Theorem 3, the control law can be computed from the expression above as

$$
\begin{aligned}
u_{f}= & \varphi_{1}(x, \phi(x)) \\
=\frac{X_{d \Sigma}^{\prime} T_{f}}{V_{\infty} \sin x_{1}}\left[-\left(-a+x_{2} \cot x_{1}\right) x_{3}-\frac{b V_{\infty} \sin 2 x_{1}}{2 X_{d \Sigma}^{\prime}}\right. & \left.\quad+\frac{\partial \pi_{3}}{\partial x_{1}} \dot{x}_{1}+\frac{\partial \pi_{3}}{\partial x_{2}} \dot{x}_{2}-\gamma_{1}\left(x_{3}-\pi_{3}\left(x_{1}, x_{2}\right)\right)\right], \\
u_{r}=\varphi_{2}(x, \phi(x)) & \frac{T_{r}}{g_{42}(x)}\left[-f_{4}(x)-g_{41}(x) \cdot \frac{\varphi_{1}(x, z)}{T_{f}}\right. \\
& \left.\quad+\frac{\partial \pi_{4}}{\partial x_{2}} \dot{x}_{2}-\gamma_{2}\left(x_{4}-\pi_{4}\left(x_{2}\right)\right)\right],
\end{aligned}
$$

where $\partial \pi_{3} / \partial x_{1}=\beta M \cos \left(x_{1}-x_{1 e}\right), \partial \pi_{3} / \partial x_{2}=0, \partial \pi_{4} / \partial x_{2}=$ $\gamma_{d}$, and $f_{4}(x), g_{41}(x), g_{42}(x)$, and $\dot{x}_{1}, \dot{x}_{2}$ are given in (5).

According to condition (H4), it is also necessary to prove boundedness of the trajectories of the closed-system with the control law $\varphi_{i}(x, \phi(x)), i=1,2$, and the off-the-manifold coordinate $z$ as given in

$$
\begin{gathered}
\dot{x}_{1}=x_{2}, \\
\dot{x}_{2}=\frac{1}{M}\left(P_{m}-D x_{2}-x_{3}-x_{4}\right), \\
\dot{x}_{3}=\left(-a+x_{2} \cot x_{1}\right) x_{3}+\frac{b V_{\infty} \sin 2 x_{1}}{X_{d \Sigma}^{\prime}} \\
+\frac{V_{\infty} \sin x_{1}}{X_{d \Sigma}^{\prime}} \cdot \frac{u_{f}}{T_{f}}, \\
\dot{x}_{4}=f_{4}(x)+g_{41}(x) \cdot \frac{u_{f}}{T_{f}}+g_{42}(x) \cdot \frac{u_{r}}{T_{r}}, \\
\dot{z}_{1}=-\gamma_{1} z_{1}, \\
\dot{z}_{2}=-\gamma_{2} z_{2} .
\end{gathered}
$$

To begin, it can be seen that clearly $x_{1} \in \mathcal{S}$ is bounded and $z_{1}$ and $z_{2}$ are exponentially decaying functions, that is, $z_{i}(t)=z_{i}(0) e^{-\gamma_{i} t}, i=1,2$, and also bounded. Substituting $x_{3}=$ $z_{1}+\pi_{3}\left(x_{1}, x_{2}\right)$ and $x_{4}=z_{2}+\pi\left(x_{2}\right)$ into the second equation of (24), using the energy function $W=(1 / 2) x_{2}^{2}+V\left(x_{1}\right)+$ $\left(z_{1}^{2}+z_{2}^{2}\right) / 2$, and using some lengthy, but straightforward, calculation yield

$$
\begin{aligned}
\dot{W} & =-\frac{D}{M} x_{2}^{2}-\frac{x_{2}\left(z_{1}+z_{2}\right)}{M}-\gamma_{1} z_{1}^{2}-\gamma_{2} z_{2}^{2} \\
& \leq-\frac{D}{M} x_{2}^{2}-\frac{1}{M}\left(\left|x_{2}\right|\left|z_{1}\right|+\left|x_{2}\right|\left|z_{2}\right|\right)-\gamma_{1} z_{1}^{2}-\gamma_{2} z_{2}^{2} \\
& \leq-\frac{(D+1)}{M} x_{2}^{2}+\Delta\left(z_{1}, z_{2}\right)
\end{aligned}
$$

with $\Delta\left(z_{1}, z_{2}\right)=-\left(\gamma_{1}+1 / 2 M\right) z_{1}^{2}-\left(\gamma_{2}+1 / 2 M\right) z_{2}^{2}$, where the first inequality follows from Young's inequality $\left(2 a b \leq c a^{2}+\right.$ $\left.(1 / c) b^{2}\right)$ that eventually leads to the last inequality. From the last inequality, with $M$ and $\gamma_{i}, i=1,2$ being positive, and $D \geq$ 0 , it can be seen that there exists a time $t_{f}$ such that $\Delta\left(z_{1}, z_{2}\right)=$ $0\left(z_{i}(t)=z_{i}(0) e^{-\gamma_{i} t}, i=1,2\right)$, for all $t \geq t_{f}$ and eventually we have $\dot{W} \leq-((D+1) / M) x_{2}^{2} \leq 0$. Therefore, there exists a ball around the operating equilibrium, strictly contained in $\mathscr{D}$ such that all trajectories starting in this set satisfy $H\left(x_{1}, x_{2}\right) \leq$ $H\left(x_{1}(0), x_{2}(0)\right)$, thus resulting in boundedness of $x_{1}$ and $x_{2}$. This implies boundedness of $\pi_{3}\left(x_{1}, x_{2}\right)$ and $\pi_{4}\left(x_{2}\right)$. Finally, boundedness of $x_{3}, x_{4}$ immediately follows from the fact that $x_{3}=z_{1}+\pi_{3}\left(x_{1}, x_{2}\right)$ and $x_{4}=z_{2}+\pi_{4}\left(x_{2}\right)$.

With the help of LaSalle's invariance principle, it can be shown that the closed-loop system at the desired equilibrium $x_{e}$ contained in the set $\mathscr{D}$ is asymptotically stable and the desired equilibrium is asymptotically stable; therefore, $x_{3}=$ $\pi\left(x_{1}, x_{2 e}\right), x_{4}=\pi_{4}\left(x_{2 e}\right)=0$, respectively. Consequently, we obtain

$$
\begin{aligned}
x_{3} & =\pi_{3}\left(x_{1}, x_{2 e}\right) \\
& =-\beta M \sin \left(x_{1}-x_{1 e}\right)+P_{m}-D x_{2 e}-x_{4 e},
\end{aligned}
$$




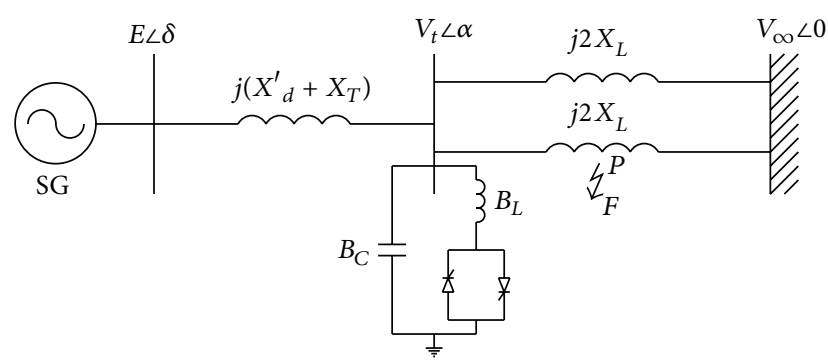

FIGURE 2: A single line diagram of SMIB with TCR-FC SVC.

and then compare (26) with the second row of (5) at the equilibrium $x_{e}$ as follows:

$$
x_{3 e}=P_{m}-D x_{2 e}-x_{4 e} .
$$

It can be obviously seen that the only solution in $\mathscr{D}$ that can make both (26) and (27) equal is $x_{1}=x_{1 e}$. This means that $x_{3}=x_{3 e}$ and both $x_{1 e}$ and $x_{3 e}$ are contained in the set $\mathscr{D}$.

Hence, boundedness of the trajectories of (24) and $\lim _{t \rightarrow+\infty} z(t)=0$ has been shown. We can establish the main result summarizing the I\&I controller design in the following proposition.

Proposition 4. The closed-loop systems in (24) with the control laws in (23) are locally asymptotically stable in $x_{e}$.

Proof. The proof of Proposition 4 is based on the arguments given above.

\section{Simulation Results}

In this section, simulation results using a combination of generator excitation and SVC controllers in a SMIB system are shown using power angle stability, and voltage and frequency regulation to point out the system stability enhancement, in particular transient stability.

Consider the single line diagram as shown in Figure 2 with the SG connected through parallel transmission lines to an infinite-bus. The SG delivers $1.0 \mathrm{pu}$. power while the terminal voltage, $V_{\text {ref }}$, is $0.9897 \mathrm{pu}$. and the infinite-bus voltage is $1.0 \mathrm{pu}$. Suppos that there is a three-phase fault occurring at point $P$, the midpoint of one of the parallel transmission lines, thereby resulting in rotor acceleration and voltage sag. Eventually, such a fault is cleared by opening and reclosing the circuit breaker at each end of the affected line.

Besides, once there is a small perturbation, particularly the mechanical input power, on the network, this causes the system trajectories, induced by the perturbation, to be confined to a limited region in a neighborhood of a nominal operating trajectory.

We are, therefore, interested in the following two questions. One is whether, after the fault is cleared from the network, the system will settle to a postfault equilibrium state. The other is whether, after the small perturbation disappears, the system can maintain stability. In this paper, the fault of interest is a temporary fault sequence and a small perturbation to mechanical power input to synchronous generators in the system is taken into account.

The physical parameters (pu.) and initial conditions $\left(\delta_{e}, \omega_{s}, P_{e e}, P_{\text {svce }}\right)$ for this power system model are given as follows:

$$
\begin{gathered}
\omega_{s}=\frac{2 \pi f \mathrm{rad}}{s}, \quad D=0.2, \quad H=5, \quad f=60 \mathrm{~Hz}, \\
T_{0}^{\prime}=4, \quad T_{r}=0.2 ; \quad V_{\infty}=1 \angle 0^{\circ}, \quad \omega=\omega_{s}, \\
X_{d}=1.1, \quad X_{d}^{\prime}=0.2, \quad X_{T}=0.1, \quad X_{L}=0.2, \\
T_{r}=0.02, \quad \delta_{e}=0.4964 \mathrm{rad}, \\
P_{e e}=P_{m}=1.0 \text { pu. }, \quad B_{L 0}=B_{C}=0.3 .
\end{gathered}
$$

The parameters of the control law are set as $\gamma_{1}=\gamma_{2}=\gamma_{3}=$ $\beta=100, \gamma_{d}=0.2$.

In this paper, two cases with different disturbances are investigated in the transient stability studies.

Case 1 (temporary fault). The system is in a prefault steady state, a fault occurs at $t_{0}=0.5 \mathrm{sec}$., the fault is isolated by opening the breaker of the faulted line at $t_{c}=0.8 \mathrm{sec}$., and the transmission line is recovered without the fault at $t_{r}=1.5 \mathrm{sec}$. Afterward, the system is in a postfault state.

Case 2 (The mechanical input power increase). The system is in the prefault state; there is a $20 \%$ perturbation in the mechanical power $P_{m}+\Delta P_{m}(t)$ where

$$
\Delta P_{m}(t)= \begin{cases}0, & \text { for } 0.0 \leq t<0.5 \mathrm{sec} . \\ 0.2 P_{m}, & \text { for } 0.5 \leq t \leq 2.5 \mathrm{sec} . \\ 0, & \text { for } t>2.5 \mathrm{sec}\end{cases}
$$

The effectiveness of the coordinated controller to improve transient stability through power angle stability with voltage and frequency regulation is determined by comparison with a Coordinated Passivation Controller (CPC) [21] and a Feedback Linearization (FBL) controller [8].

From our simulation results, it can be concluded that with the help of the proposed controller in both cases, Figures 3-6 indicate the transient behavior of the power system using the proposed controller. Evidently, transient stability is enhanced as compared to the FBL controller and CPC. The closed-loop system is transiently and asymptotically stable even when a large disturbance (temporary fault) occurs on the network and there is a mechanical input increase.

It is clear from Case 1 that Figure 3 shows time trajectories of power angle $\delta$, SG relative speed (frequency) $\omega-\omega_{s}$, transient voltage $(E)$, and SVC susceptance of three controllers. It can easily be seen that the power angles $(\delta)$, the SG relative speeds $\left(\omega-\omega_{s}\right)$, transient voltages $(E)$, and SVC susceptance of the three controllers restore to the prefault values $\left(\delta \rightarrow \delta_{e}, \omega-\omega_{s} \rightarrow 0, E \rightarrow E_{e}, B_{L} \rightarrow B_{L 0}\right)$, respectively; however, time histories of each controller have the different damping levels. Clearly, the proposed controller provides better transient response performance in terms of 


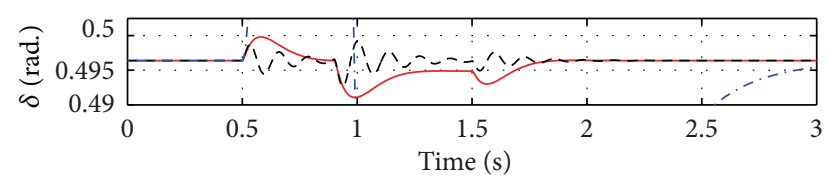

(a)

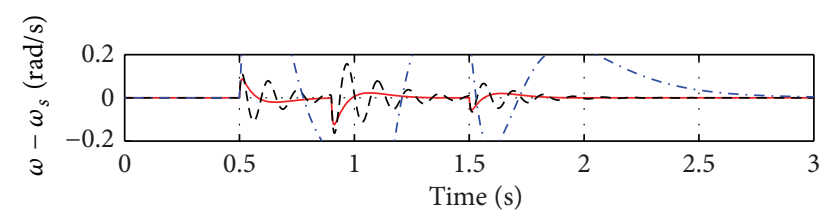

(b)

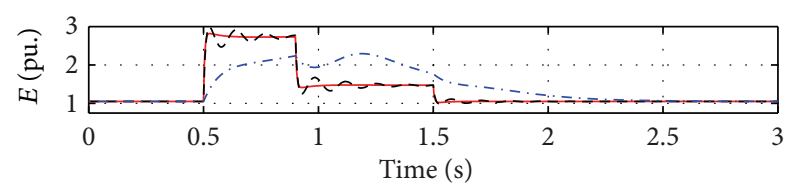

- I\&I

-- CPC

-.. FBL

(c)

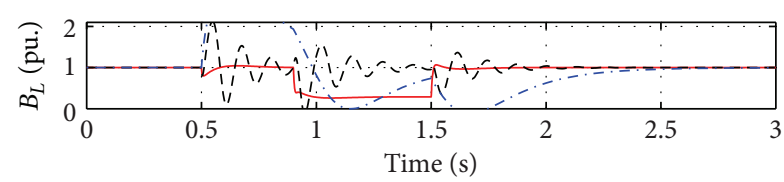

(d)

FIgURE 3: Case 1: time histories of power angle $(\delta)$, relative speed $\left(\omega-\omega_{s}\right)$, transient voltage $(E)$, and susceptance of SVC $\left(B_{L}\right)$. Solid: I\&I controller; dashed: Coordinated Passivation Controller (CPC); dash-dotted: FBL.

reduced overshoot and faster reduction of oscillations except for the power angles $(\delta)$ of both cases.

Figure 4 provides time trajectories of active electrical power $P_{e}$ and terminal voltage $V_{t}$ of three controllers settling to the prefault steady state $\left(P_{e} \rightarrow P_{m}\right.$ and $\left.V_{t} \rightarrow V_{\text {ref }}\right)$. Further, power and voltage regulation of three controllers are simultaneously achieved but when compared with the $\mathrm{CPC}$ and FBL controllers, the proposed controller can more quickly damp the oscillations and return to the prefault values almost without sustained oscillations. Also, Figure 4 illustrates the off-the-manifold coordinates $z_{1}$ and $z_{2}$ showing the manifold $\mathscr{M}$ implicitly described by $\phi(x)=0$ as expected.

Similar to temporary fault case, it is evident from Case 2 that in Figure 5 time trajectories of power angle $\delta$, SG relative speed (frequency) $\omega-\omega_{s}$, transient voltage $(E)$, and SVC susceptance of the proposed controller settle to the prefault steady-state values more quickly. In comparison with both FBL controller and CPC, the I\&I controller provides obviously the fastest reduction of oscillation as well as the smallest overshoot except for power angle and SG relative speed. In a similar way, Figure 6 shows time trajectories of active power and terminal voltage of three controllers along with the off-the-manifold coordinates $z_{1}$ and $z_{3}$ getting back

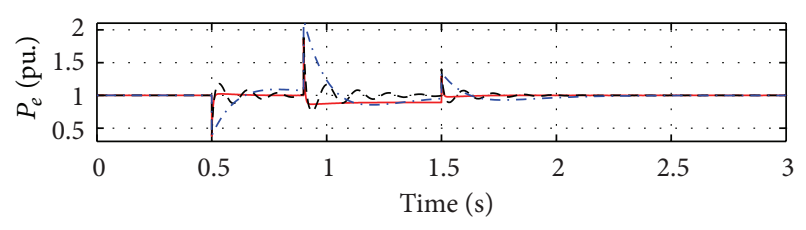

(a)

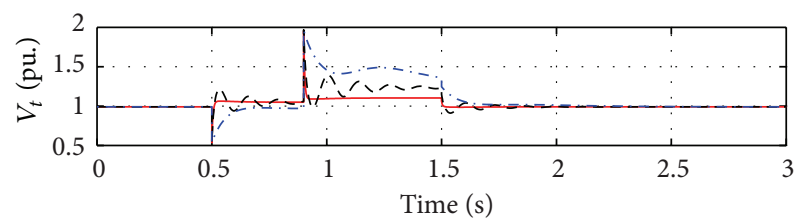

(b)

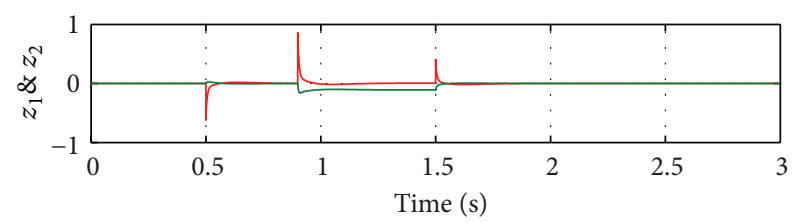

(c)

Figure 4: Case 1: time histories of active power $\left(P_{e}\right)$ and terminal voltage $\left(V_{t}\right)$ and the off-the-line manifold coordinates $z_{1}, z_{2}$. Solid: I\&I controller; dashed: Coordinated Passivation Controller (CPC), dash-dotted: FBL.

to the prefault steady states $\left(P_{e} \rightarrow P_{m}, V_{t} \rightarrow V_{\text {ref }}, z_{1} \rightarrow 0\right.$, and $\left.z_{2} \rightarrow 0\right)$ after the small perturbation vanishes.

From simulation results above, it can be overall concluded that, regardless of the steady-state operating point of the system, the I\&I control design technique is capable of not only achieving the expected performance requirements (1)(2) mentioned above but also accomplishing better dynamic performance (improved transient responses for the closedloop system) after an occurrence of a temporary fault or a small perturbation.

\section{Conclusion}

In this paper, we have shown that nonlinear coordinated generator excitation and SVC control in SMIB power systems can be effectively used to enhance the transient stability of power systems after the occurrence of a large disturbance and a small perturbation. Using a nonlinear system model and the I\&I design methodology, simulation results have demonstrated the effectiveness of the proposed controller capable of achieving closed-loop system transient stability, accomplishing power angle stability along with voltage and frequency regulation, and providing improved transient response over the existing nonlinear controllers, that is, the Feedback Linearization (FBL) and the coordinated passivation controller. Finally, it can be also seen that the proposed controller is dependent on all measurable states which are often monitored in practice; thus, our results of this work are of practical significance and applicable value. 


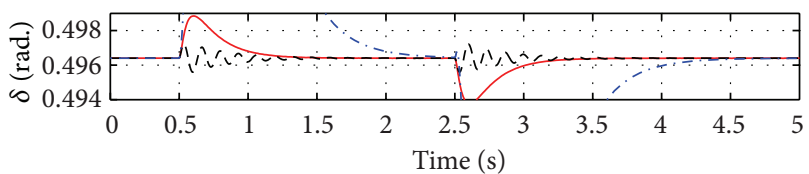

(a)

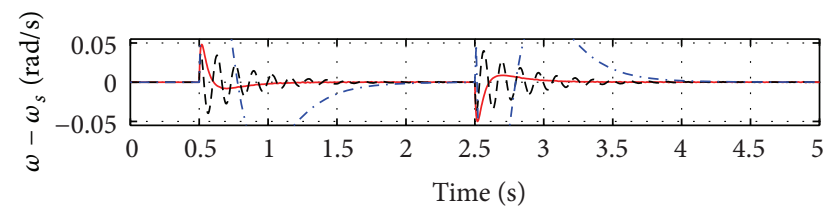

(b)

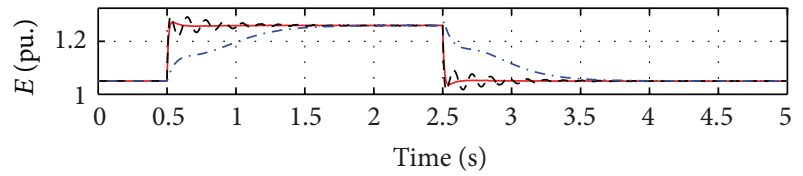

- I\&I

--- CPC

-.. FBL

(c)

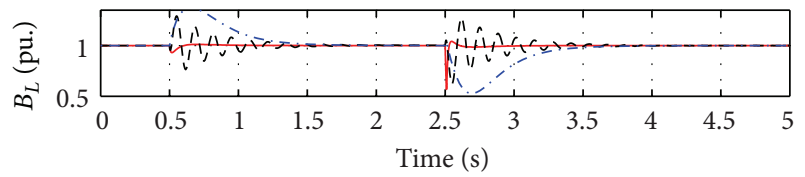

(d)

FIgURE 5: Case 2: time histories of power angle $(\delta)$, relative speed $\left(\omega-\omega_{s}\right)$, transient voltage $(E)$, and susceptance of SVC $\left(B_{L}\right)$. Solid: I\&I controller; dashed: Coordinated Passivation Controller (CPC); dash-dotted: FBL.

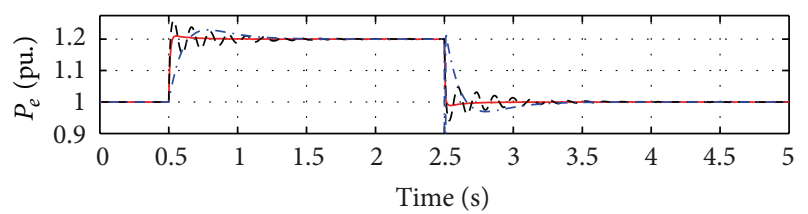

(a)

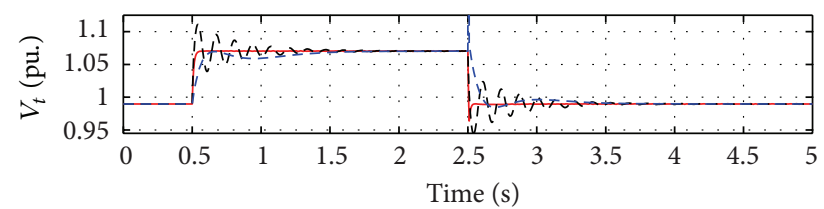

(b)

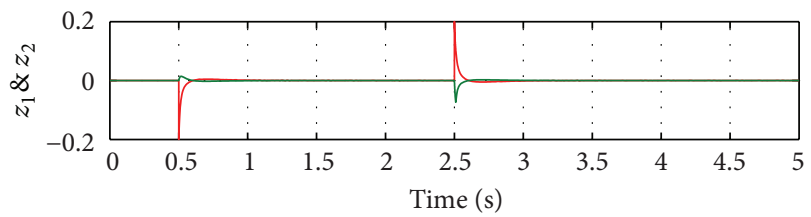

(c)

Figure 6: Case 2: time histories of active power $\left(P_{e}\right)$ and terminal voltage $\left(V_{t}\right)$ and the off-the-line manifold coordinates $z_{1}, z_{2}$. Solid: I\&I controller; dashed: Coordinated Passivation Controller (CPC); dash-dotted: FBL.

\section{Appendices}

\section{A. Feedback Linearization Controller [8]}

In order to design a nonlinear coordinated controller based on feedback linearization scheme used to compare with the proposed controller, let us define the output as

$$
\begin{gathered}
y_{1}=h_{1}(x)=P_{e}-P_{m}=\Delta P_{e} \\
y_{2}=h_{2}(x)=P_{\mathrm{svc}}-P_{\mathrm{svce}}=\Delta P_{\mathrm{svc}} ;
\end{gathered}
$$

then the power system (5) is a three-input three-output nonlinear system which can be written as

$$
\begin{gathered}
\dot{x}=f(x)+g_{1}(x) \frac{u_{f}}{T_{f}}+g_{2}(x) \frac{u_{r}}{T_{r}}, \\
y=h(x),
\end{gathered}
$$

where

$$
x=\left[\begin{array}{l}
x_{1} \\
x_{2} \\
x_{3} \\
x_{4}
\end{array}\right], \quad f(x)=\left[\begin{array}{l}
f_{1}(x) \\
f_{2}(x) \\
f_{3}(x) \\
f_{4}(x)
\end{array}\right],
$$

$$
\begin{gathered}
g_{1}(x)=\left[\begin{array}{c}
0 \\
0 \\
g_{31}(x) \\
g_{41}(x)
\end{array}\right]=\left[\begin{array}{c}
0 \\
0 \\
\widetilde{M}\left(x_{1}, x_{3}, x_{4}\right) g_{31}(x)
\end{array}\right], \\
g_{2}(x)=\left[\begin{array}{c}
0 \\
0 \\
0 \\
g_{42}(x)
\end{array}\right]=\left[\begin{array}{c}
0 \\
0 \\
0 \\
-\widetilde{N}\left(x_{1}, x_{3}, x_{4}\right)
\end{array}\right] .
\end{gathered}
$$

With the help of the differential geometry theory, we have that the relative degree of the nonlinear system (A.2) at $x_{e}$ becomes $r=1+1=2<4$; therefore, based on the stability theory of the zero dynamics, we select the change of coordinates as follows:

$$
\Phi(x)=\left[\begin{array}{c}
\widetilde{z}_{1} \\
\tilde{z}_{2} \\
\tilde{z}_{3} \\
\tilde{z}_{4}
\end{array}\right]=\left[\begin{array}{c}
x_{1} \\
x_{2} \\
h_{1}(x) \\
h_{2}(x)
\end{array}\right],
$$

and we assume that the Jacobian matrix of $\Phi(x)$ at $x_{e}$ is nonsingular $\left(\operatorname{det}\left((\partial \Phi(x) / \partial x)_{\left.\right|_{x}}\right) \neq 0\right)$. Further, it is easy to express the nonlinear system (A.2) as

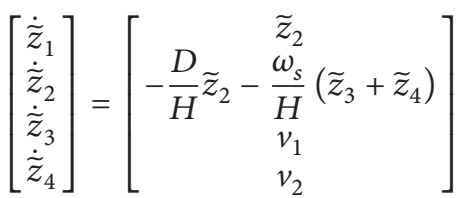




$$
\begin{aligned}
& =\left[\begin{array}{cccc}
0 & 1 & 0 & 0 \\
0 & -\frac{D}{H} & -\frac{\omega_{s}}{H} & -\frac{\omega_{s}}{H} \\
0 & 0 & 0 & 0 \\
0 & 0 & 0 & 0
\end{array}\right]\left[\begin{array}{c}
\widetilde{z}_{1} \\
\widetilde{z}_{2} \\
\widetilde{z}_{3} \\
\widetilde{z}_{4}
\end{array}\right]+\left[\begin{array}{ll}
0 & 0 \\
0 & 0 \\
1 & 0 \\
0 & 1
\end{array}\right]\left[\begin{array}{l}
v_{1} \\
v_{2}
\end{array}\right] \\
& =A \widetilde{z}+B v .
\end{aligned}
$$

For the linear system (A.5), the linear optimal law $v$ can be obtained as

$$
v(\widetilde{z})=\left[\begin{array}{l}
v_{1}(\widetilde{z}) \\
v_{2}(\widetilde{z})
\end{array}\right]=-B^{T} P \widetilde{z},
$$

where $P$ is the positive definite matrix satisfying the Riccati equation for linear systems (A.5). Hence, it is easy to obtain the nonlinear coordinated and optimal control law based on feedback linearization methodology as follows:

$$
\left[\begin{array}{l}
\frac{u_{f}}{T_{f}} \\
\frac{u_{r}}{T_{r}}
\end{array}\right]=\left[\begin{array}{c}
-\frac{f_{3}(x)-v_{1}(x)}{g_{31}(x)} \\
-\frac{g_{41}(x)\left(f_{3}(x)-v_{1}(x)\right)}{g_{31}(x) g_{42}(x)}-\frac{f_{4}(x)-v_{2}(x)}{g_{42}(x)}
\end{array}\right] .
$$

\section{B. Coordinated Passivation Controller [21]}

For simplicity, let us define the state variable by $\tilde{x}_{1}=\delta-\delta_{e}$, $\tilde{x}_{2}=\omega-\omega_{s}, \widetilde{x}_{3}=P_{e}-P_{m}, y=P_{\text {svc }}-P_{\text {svce }}$; therefore, the power system considered in (5) is output strictly passive under the following Coordination Passivation Controller

$$
\begin{aligned}
& \frac{V_{\infty} \sin x_{1}}{X_{d \Sigma}^{\prime} T_{f}} u_{f}= \frac{\tilde{x}_{2}+c_{1} \tilde{x}_{1}}{M}-c_{3}\left(\tilde{x}_{3}-\alpha\left(\tilde{x}_{1}, \tilde{x}_{2}\right)\right) \\
&-\left(-a+\tilde{x}_{2} \cot \left(\tilde{x}_{1}+\delta_{e}\right)\right)\left(\tilde{x}_{3}+P_{m}\right) \\
&-\frac{b V_{\infty} \sin 2\left(\tilde{x}_{1}+\delta_{e}\right)}{2 X_{d \Sigma}^{\prime}}+\frac{\partial \alpha}{\partial \tilde{x}_{1}} \tilde{x}_{2}+\frac{\partial \alpha}{\partial \tilde{x}_{2}} \dot{\tilde{x}}_{2} \\
& g_{42}(x) \frac{u_{r}}{T_{r}}=-f_{4}(x, y)-g_{41}(x, y) \frac{u_{f}}{T_{f}}-\frac{\tilde{x}_{2}+c_{1} \tilde{x}_{1}}{M} y+v
\end{aligned}
$$

where $\alpha\left(\tilde{x}_{1}, \tilde{x}_{2}\right)=-(1 / M)\left(c_{2}\left(\tilde{x}_{2}+c_{1} \tilde{x}_{1}\right)+\tilde{x}_{1}+\left(c_{1}-D / H\right) \tilde{x}_{2}\right)$, and in this paper tuning parameters are chosen as $c_{1}=c_{2}=$ $c_{3}=20$. Further, if $v=-\widetilde{\beta} y(\tilde{\beta}>0)$ and $c_{1}+c_{2}+c_{3}>(D / H)$, the closed-loop system is asymptotically stable.

\section{Conflict of Interests}

The author declares that there is no conflict of interests regarding the publication of this paper.

\section{References}

[1] N. G. Hingorani and L. Gyugyi, Understanding FACTS: Concepts and Technology of Flexible AC Transmission Systems, IEEE Press, Piscataway, NJ, USA, 1999.
[2] Y. N. Song and A. T. John, Flexible AC Transmission Systems (FACTS), vol. 30 of IEE Power and Energy Series, 1999.

[3] E. Z. Zhou, "Application of static Var compensators to increase power system damping," IEEE Transactions on Power Systems, vol. 8, no. 2, pp. 655-661, 1993.

[4] K. T. Law, D. J. Hill, and N. R. Godfrey, "Robust controller structure for coordinated power system voltage regulator and stabilizer design," IEEE Transactions on Control Systems Technology, vol. 2, no. 3, pp. 220-232, 1994.

[5] L. Cong, Y. Wang, and D. J. Hill, "Transient stability and voltage regulation enhancement via coordinated control of generator excitation and SVC," International Journal of Electrical Power \& Energy Systems, vol. 27, no. 2, pp. 121-130, 2005.

[6] L. Cong, Y. Wang, and D. J. Hill, "Co-ordinated control design of generator excitation and SVC for transient stability and voltage regulation enhancement of multi-machine power systems," International Journal of Robust and Nonlinear Control, vol. 14, no. 9-10, pp. 789-805, 2004.

[7] K. R. Padiyar and R. K. Varma, "Damping torque analysis of static VAR system controllers," IEEE Transactions on Power Systems, vol. 6, no. 2, pp. 458-465, 1991.

[8] Q. Lu, Y. Sun, and S. Mei, Nonlinear Control Systems and Power System Dynamics, Kluwer Academic, New York, NY, USA, 2001.

[9] Y. Ruan and J. Wang, "The coordinated control of SVC and excitation of generators in power systems with nonlinear loads," International Journal of Electrical Power \& Energy Systems, vol. 27, no. 8, pp. 550-555, 2005.

[10] Y. Wang, H. Chen, and R. Zhou, "A nonlinear controller design for SVC to improve power system voltage stability," International Journal of Electrical Power \& Energy Systems, vol. 22, no. 7, pp. 463-470, 2000.

[11] R. Yan, Z. Y. Dong, T. K. Saha, and J. Ma, "Nonlinear robust adaptive SVC controller design for power systems," in Proceedings of the IEEE Power and Energy Society General Meeting: Conversion and Delivery of Electrical Energy in the 21st Century, pp. 1-7, Pittsburgh, Pa, USA, July 2008.

[12] L.-Y. Sun, S. Tong, and Y. Liu, "Adaptive backstepping sliding mode $H_{\infty}$ control of static Var compensator," IEEE Transactions on Control Systems Technology, vol. 19, no. 5, pp. 1178-1185, 2011.

[13] A. Kanchanaharuthai, "Immersion and invariance-based nonlinear controller for a power system with the excitation and STATCOM," Rangsit Journal of Arts and Sciences, vol. 2, no. 2, pp. 151-160, 2012.

[14] N. S. Manjarekar, R. N. Banavar, and R. Ortega, "Nonlinear control synthesis for asymptotic stabilization of the swing equation using a controllable series capacitor via immersion and invariance," in Proceedings of the 47th IEEE Conference on Decision and Control (CDC '08), pp. 2493-2498, Cancún, Mexico, December 2008.

[15] M. Krstic, I. Kanellakopoulos, and P. Kokotivic, Nonlinear and Adaptive Control Design, John Willey \& Sons, New York, NY, USA, 1995.

[16] Y. Wan and J. Zhao, "Extended backstepping method for single-machine infinite-bus power systems with SMES," IEEE Transactions on Control Systems Technology, vol. 21, no. 3, pp. 915-923, 2013.

[17] A. Astolfi and R. Ortega, "Immersion and invariance: a new tool for stabilization and adaptive control of nonlinear systems," IEEE Transactions on Automatic Control, vol. 48, no. 4, pp. 590606, 2003.

[18] A. Astolfi, D. Karagiannis, and R. Oreta, Nonlinear and Adaptive Control Design with Applications, Springer, London, UK, 2007. 
[19] W. Dib, G. Kenné, and F. Lamnabhi-Lagarrigue, "An application of immersion and invariance to transient stability and voltage regulation of power systems with unknown mechanical power," in Proceedings of the 48th IEEE Conference on Decision and Control held jointly with 28th Chinese Control Conference (CDC/CCC '09), pp. 7837-7842, Shanghai, China, December 2009.

[20] W. Dib, R. Ortega, A. Astolfi, and D. Hill, "Improving transient stability of multi-machine power systems: synchronization via immersion of a pendular system," in Proceedings of the American Control Conference (ACC '11), pp. 1408-1413, San Francisco, Calif, USA, July 2011.

[21] H. Chen, H.-B. Ji, B. Wang, and H.-S. Xi, "Coordinated passivation techniques for the dual-excited and steam-valving control of synchronous generators," IEE Proceedings-Control Theory and Applications, vol. 153, no. 1, pp. 69-73, 2006. 


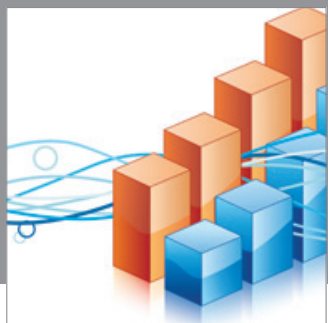

Advances in

Operations Research

mansans

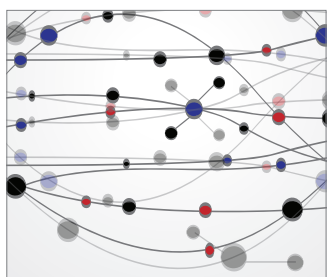

The Scientific World Journal
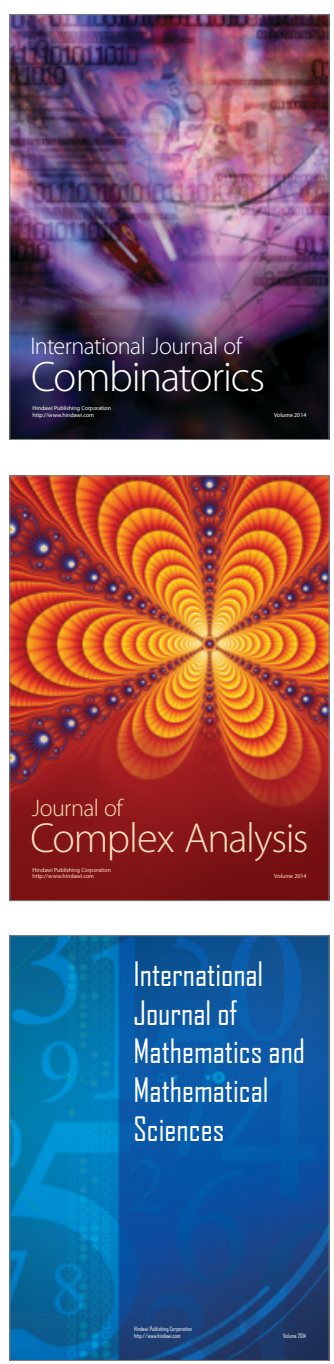
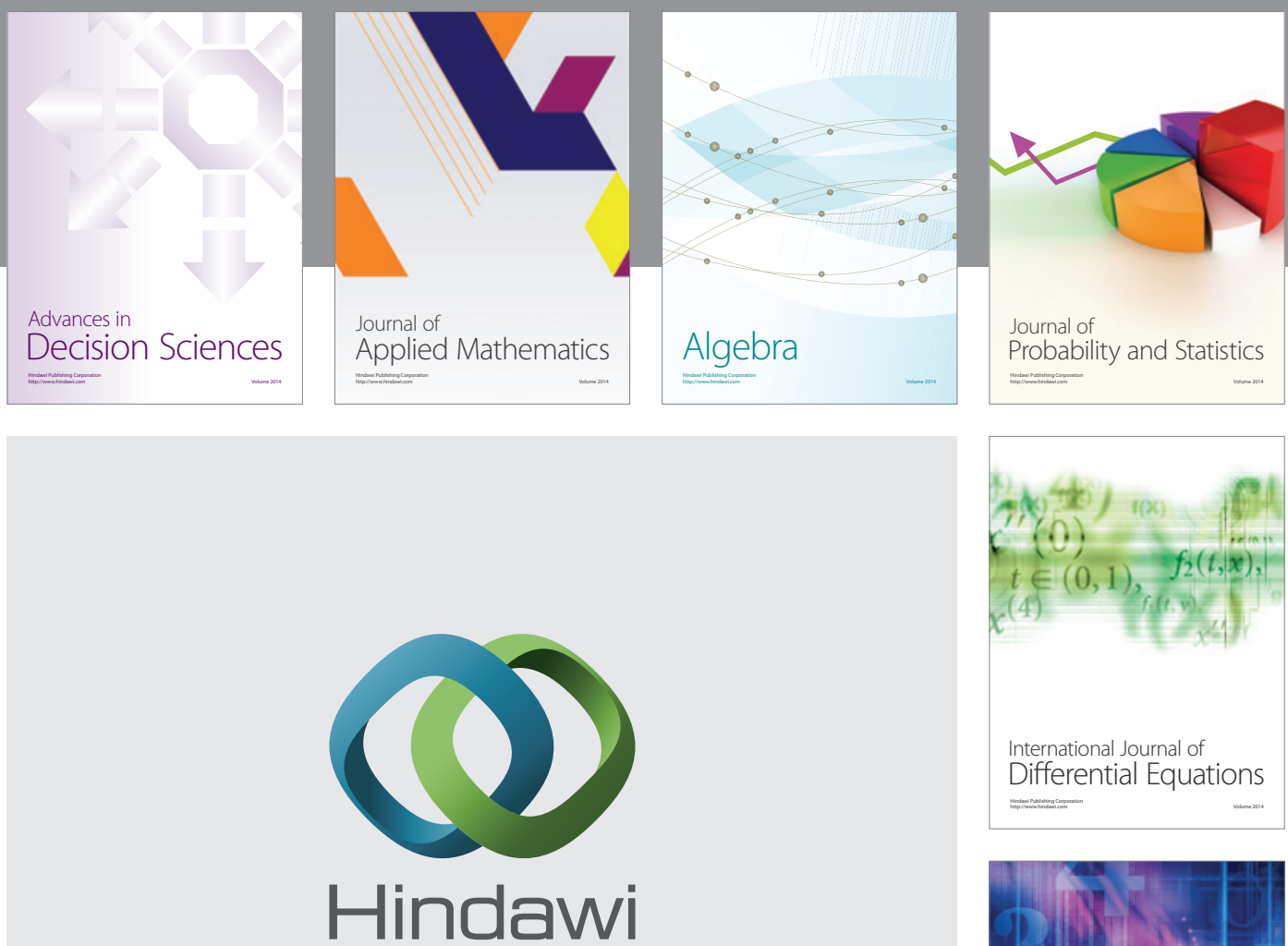

Submit your manuscripts at http://www.hindawi.com
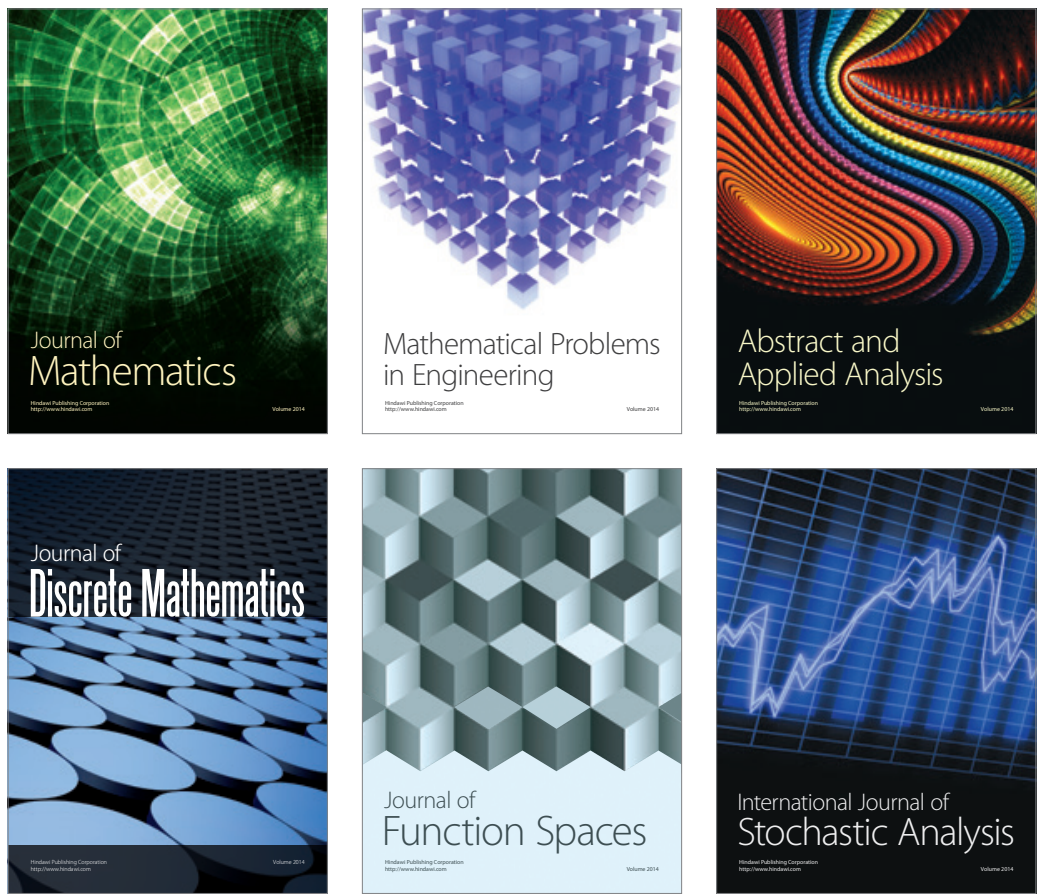

Journal of

Function Spaces

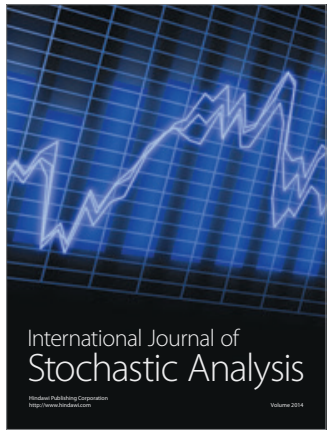

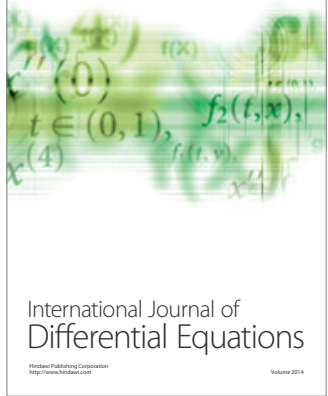
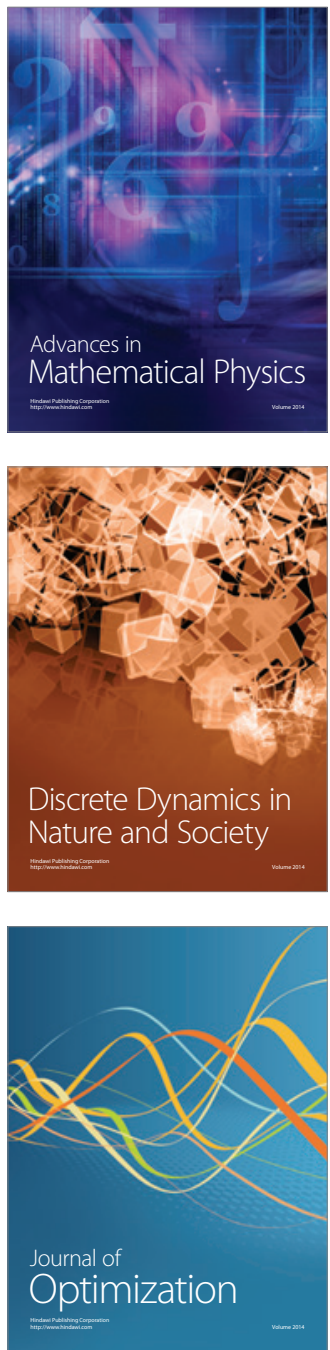